\title{
Estimation of Non-WSSUS Channel for OFDM Systems in High Speed Railway Environment Using Compressive Sensing*
}

\author{
Chen Wang, Yong Fang, Zhi-Chao Sheng \\ School of Communication and Information Engineering, Shanghai University, Shanghai, China \\ Email: cwangsh@shu.edu.cn,yfang@staff.shu.edu.cn, kebon22@shu.edu.cn
}

Received July 2013

\begin{abstract}
Non Wide Sense Stationary Uncorrelated Scattering (Non-WSSUS) is one of characteristics for high-speed railway wireless channels. In this paper, estimation of Non-WSSUS Channel for OFDM Systems is considered by using Compressive Sensing (CS) method. Given sufficiently wide transmission bandwidth, wireless channels encountered here tend to exhibit a sparse multipath structure. Then a sparse Non-WSSUS channel estimation approach is proposed based on the delay-Doppler-spread function representation of the channel. This approach includes two steps. First, the delay-Doppler-spread function is estimated by the Compressive Sensing (CS) method utilizing the delay-Doppler basis. Then, the channel is tracked by a reduced order Kalman filter in the sparse delay-Doppler domain, and then estimated sequentially. Simulation results under LTE-R standard demonstrate that the proposed algorithm significantly improves the performance of channel estimation, comparing with the conventional Least Square (LS) and regular CS methods.
\end{abstract}

Keywords: OFDM; Non-WSSUS Channel Estimation; Compressive Sensing (CS); Kalman Filter; LTE-R

\section{Introduction}

In recent several years, High-Speed Railway (HSR) in China has made great progress and attracted the world's attention [1]. The new speed record of high speed train is at $486.1 \mathrm{~km} / \mathrm{h}$ [2]. The broadband wireless access on HSRs, also known as train-ground communication has become a hot issue. To develop high quality service wireless communications that meet the demand of next-generation broadband wireless access for high-speed railway has become an urgent problem. It has been shown that the communication quality of the existing wireless network of HSRs is quite poor, where a high rate of dropped calls and low data rate are observed [3]. LTE-R [4,5] basing on OFDM is commonly considered as a promising candidate to provide high quality of service. Since the serious time and frequency selective fading, which affects the OFDM symbol at the physical layer greatly impacts system OFDM performance, the estimation and compensation of the channel variation for each OFDM symbol is crucial [6].

In the wireless communication, one of the most practical assumptions about the wireless channel is that of

*This work was supported by the National Science Foundation of China (61271213, 61132004, 60972056).
Wide Sense Stationary Uncorrelated Scattering, (WSSUS). Existing fast fading channel estimation methods most generalized stationary uncorrelated scattering (WSSUS) as a precondition [7], or satisfied some certain channel statistical characteristics, e.g. Jakes' channels [8]. However, this assumption is no longer valid when the transceivers operate in the high speed railway environment. Because for the HSR channel, the transceiver encounters different channel conditions and the train runs across the scenarios so rapidly [3]. This condition provokes the multipath arrivals associated with surface scattering fluctuate rapidly over time, in the sense that the channel gain, the arrival time, and the Doppler shifts of each arrival all change dynamically [9], as a result the channel is NonWSSUS. The study of statistical characteristics of NonWSSUS has drawn much attention of researchers $[10,11]$. But in order to perform OFDM channel estimation, the channel impulse response (CIR) is needed to be estimated.

Apart from the fact that HSR channel is Non-WSSUS, it is also sparse. For one thing, numerous experimental studies undertaken by various researchers in the recent have shown that wireless channels associated with a number of scattering environments tend to exhibit sparse structures at large bandwidths [12]. For another, compared 
with urban and indoor conditions, the specular LOS component is much stronger. Thus, the channel is generally lower density scattered [3], which enhances the sparse structures of the HSR channel.

According to the characteristics of HSR channel mentioned above, a Non-WSSUS channel estimation approach is developed in this paper. It utilizes the channel delayDoppler-spread representation to accommodate the NonWSSUS of the CIR. This approach includes two steps. In the first step, it utilizes the delay-Doppler shifts basis to estimate the sparse HSR channel. In the second step, the channel is tracked by a reduced order Kalman filter in the sparse delay-Doppler shift domain, and then recovered by CS method sequentially. One can, in turn, use the detected pilots to perform a sequential approach for channel estimation and data recovery. Our approach is not limited by certain statistical characteristics.

This paper is organized as follows: Section 2 introduces the Non-WSSUS channel and the OFDM system models. Section 3 explains the estimation of the sparse channel delay-Doppler-spread in the OFDM system. Section 4 describes the sparse dynamic model for the NonWSSUS HSR channel, and then our proposed approach for estimating Non-WSSUS channel is derived. Section 5 presents the simulations results which validate our approach. Finally, our conclusions are presented in Section 6.

\section{Non-WSSUS Channel and OFDM System Models}

\subsection{Non-WSSUS Channel Models}

There are many equivalent ways of characterizing LTV systems. We use the delay-Doppler-spread function $C(v$, $\tau$ ) for channel characterization [13,14]. The time-varying frequency response $\mathrm{H}(t, f)$ and the delay-Doppler spreading function constitute a two-dimensional Fourier transform pair. It is defined by

$$
\mathrm{H}(t, f)=\iint_{\mathbb{R}^{2}} \mathrm{C}(v, \tau) \mathrm{e}^{j 2 \pi v t} \mathrm{e}^{-j 2 \pi \tau f} d v d \tau
$$

The quantity $\mathrm{C}(v, \tau) d v d \tau$ is the contribution to from a scatterer at delay and Doppler. The discrete representation of Equation (1) is given by [12],

$$
\mathrm{H}(t, f)=\sum_{n=1}^{N_{p}} \alpha_{n} \mathrm{e}^{-j 2 \pi \tau_{n} f} \mathrm{e}^{2 \pi v_{n} t}
$$

which represents signal propagation over $N_{p}$ paths. In another word, there are $N_{p}$ pairs, corresponding to distinct scatterers at different delay and Doppler. We assume that the channel is maximally spread in the delay and Doppler space, $\tau_{n} \in\left[0, \tau_{\max }\right]$ and $v_{n} \in\left[0, v_{\text {max }}\right]$. It has been shown that if $\alpha_{n}$ (the discrete coefficients of $\mathrm{C}(v, \tau))$ is a function of discrete time $n: \alpha_{n}(n)$, then channel is not WSS anymore [13]. If the matrix repre- sentation of $\alpha_{n}$ is not diagonal, then channel is not US, which will be further demonstrated in Section 3 .

\subsection{OFDM System Models}

In OFDM systems, the output symbol of the transmitter at time $n$ is given by the $N$ point complex modulation sequence : $x_{n}=\frac{1}{\sqrt{N}} \sum_{k=0}^{N-1} X_{k} \mathrm{e}^{\frac{j 2 \pi n k}{N}}$, where $X_{k}$ is the data symbol.

The orthogonal short-time Fourier (STF) basis waveforms $\left\{g\left(t-n T_{0}\right) \mathrm{e}^{j 2 \pi m W_{0} t}\right)$, as a generalization of OFDM signaling to counteract the time selectivity of doublyselective channels are used [14]. The parameter

$T_{0} \in\left[\tau_{\max }, 1 / v_{\max }\right]$ and $W_{0} \in\left[v_{\max }, 1 / \tau_{\max }\right]$ correspond to the time and frequency separation of the STF basis, and are chosen so that $T_{0} W_{0}=1$ (which gives rise to an orthogonal STF basis).

\section{Estimation of the OFDM Sparse Channel Delay-Doppler-Spread}

\subsection{Delay-Doppler-Spread Representation}

Consider signaling over wireless channels using symbols of duration $T$ and (two-sided) bandwidth $W, x(t)=0$ $\forall t \notin[0, T]$ and $X(f)=0, \forall f \notin[-W / 2, W / 2]$, thereby giving rise to a temporal signal space of dimension $N_{0}=T W \quad[12]$.

So the maximum number of resolvable delays, $L=\left\lceil W \tau_{\max }\right\rceil+1$ and the maximum number of resolvable Doppler shift, $K=\left\lceil T v_{\max } / 2\right\rceil$. The matrix representation of delay-Doppler-spread $\alpha_{n}$ [15] is as

$$
\mathrm{H}_{\alpha}=\left[\begin{array}{cccc}
h_{0,-K} & h_{0,-K+1} & \cdots & h_{0, K} \\
h_{1,-K} & h_{1,-K+1} & \cdots & h_{1, K} \\
\vdots & \vdots & & \vdots \\
h_{L-1,-K} & h_{L-1,-K+1} & \cdots & h_{L-1, K}
\end{array}\right]
$$

Now recall from Section 2 that the time-varying frequency response (Equation (2)). The virtual representation of a doubly-selective channel therefore implies that $\mathrm{H}(t, f) \approx \sum_{l=0}^{L-1} \sum_{k=-K}^{K} \alpha \mathrm{e}^{j 2 \pi \frac{k}{T} t} \mathrm{e}^{-j 2 \pi \frac{l}{W} f}$. Consequently, the STF channel coefficients $\left\{\mathrm{H}_{n, m}\right\}$ can be written as

$$
\mathrm{H}_{n, m}=\sum_{l=0}^{L-1} \sum_{k=-K}^{K} \alpha \mathrm{e}^{j 2 \pi \frac{k}{N_{t}} n} \mathrm{e}^{-j 2 \pi \frac{l}{N_{f}} m}=\mathrm{u}_{f, m}^{\prime} \mathrm{H}_{\alpha} \mathrm{u}_{t, n}
$$

Where $N_{t}=T / T_{0} \quad N_{f}=W / W_{0}$

$$
N_{0}=N_{t} N_{f} \quad W_{N_{0}}=\mathrm{e}^{-j \frac{2 \pi}{N_{0}}}
$$

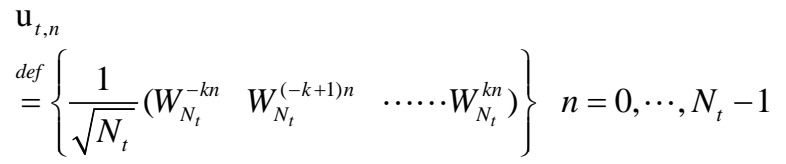




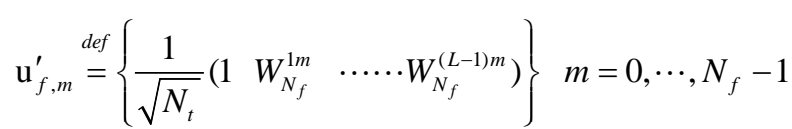

It is straightforward to see that if $\mathrm{H}_{\alpha}$ is not diagonal, then the lth scatterer will be interfered by other scatterers, leading to a correlated scatterer.

\subsection{Using CS to Estimate the Sparse Channel Delay-Doppler-Spread}

By sampling pilot symbols uniformly at random (without replacement) from the whole temporal signal space of dimension $N_{0}$, the D-sparse (the number of nonzero elements of delay-Doppler-spread function is D) channel delay-Doppler-spread estimation will have the following equation,

$$
\mathrm{H}_{p}=\mathrm{U}_{p} \operatorname{vec}\left(\mathrm{H}_{\alpha}\right)+w_{t}
$$

where $w_{t} \sim N\left(0, \sigma_{o b s}^{2} I\right), \quad \mathrm{H}_{p}=\frac{y_{p} \text { (receive pilots) }}{x_{p} \text { (transmit pilots) }}$, $\mathrm{U}_{p}=\left\{\sqrt{\varepsilon / N_{p}}\left(\mathrm{u}_{t, n}^{\prime} \otimes \mathrm{u}_{f, m}^{\prime}\right):(n, m) \in\right.$ pilots $\}, \quad \varepsilon$ is the system transmit energy budget, and $w_{t}$ is the channel observation noise.

Since $\mathrm{H}_{\alpha}$ is sparse, it is can be recovered form $\mathrm{H}_{p}$ by various CS methods (here we use OMP and CoSaMP). Then the CIR can be built according to Equation (4).

\section{Estimation of Non-WSSUS Channel}

\subsection{Non-WSSUS Channel Model}

Since the HSR channel is determined by its delayDoppler-spread representation, we just need to discuss the dynamic model for delay-Doppler-spread function. For the currently non-zero coefficients of $\mathrm{H}_{\alpha}(n)$ at discrete time $n$, we assume a spatially i.i.d. Gaussian random walk model, with noise variance $\sigma_{\text {sys }}$. The initial $\mathrm{H}_{\alpha}(0)$ is estimated by the CS sparse channel delayDoppler-spread estimation method mentioned in Section 3. $\mathrm{H}_{\alpha}(0)$ is assumed to be generated from a zero mean Gaussian with variance $\sigma_{D}^{2}\left(\sigma_{D}^{2}=\sqrt{1 / D}\right)$, and the nonzero element is randomly selected.

Let $\mathrm{T}_{n}$ denote the support set of $\mathrm{H}_{\alpha}(n)$, i.e. the set of its nonzero coordinates, and let $S_{n}=\operatorname{size}\left(\mathrm{H}_{\alpha}(n)\right)$. In other words, $\mathrm{T}_{n}=\left[i_{1}, i_{2} \ldots i_{S_{n}}\right]$ where $i_{k}$ are the non-zero coordinates of $\mathrm{H}_{\alpha}(n)$. Thus, under this assumption we have the dynamic model shown in Table 1.

Table 1. Dynamic model for sparse delay-Doppler-spread.

\begin{tabular}{c}
\hline$H_{\alpha}(0)$ is generated from the D-sparse channel with randomly selected \\
nonzero elements \\
\hline$\left(\mathrm{H}_{\alpha}(n)\right)_{i}=\left(\mathrm{H}_{\alpha}(n-1)\right)_{i}+\left(v_{n}\right)_{i}, \quad\left(v_{n}\right)_{i} \sim N\left(0, \sigma_{s y s}^{2}\right)$ if $i \in T_{n}, i \in T_{n-1}$ \\
$\left(\mathrm{H}_{\alpha}(n)\right)_{i}=\left(\mathrm{H}_{\alpha}(n-1)\right)_{i}+\left(v_{n}\right)_{i},\left(v_{n}\right)_{i}, N\left(0, \sigma_{D}^{2}\right)$ if $i \in T_{n}, i \notin T_{n-1}$ \\
$\left(\mathrm{H}_{\alpha}(n)\right)_{i}=\left(\mathrm{H}_{\alpha}(n-1)\right)_{i}$ if $i \notin T$ \\
\hline
\end{tabular}

\subsection{Estimation of Non-WSSUS Channel}

Combining observation equation (Equation (5)) and state equation (Table 1) together, we get Non-WSSUS channel representation by utilizing the sparse delay-Dopplerspread function. This dynamic model of $\mathrm{H}_{\alpha}(n)$ can be tracked by Kalman filter, and then the CIR of NonWSSUS channel can be recovered, as a result, the NonWSSUS channel will be estimated sequentially. Since the delay-Doppler-spread is sparse, KF-CS method is employed to solve the dynamic sparse channel problem. The proof of the convergence of KF-CS and more details can be found in [16,17]. Take the KF-CS algorithm into account, our proposed two-step approach is summarized in Table 2.

\section{Simulation}

This approach was tested and compared according to the LTE-R standard. The channel parameters are: Channel parameters: $\tau_{\max }=10 \mu \mathrm{s}$, and the speed of the high speed train is $500 \mathrm{~km} / \mathrm{h}$ leading to $v_{\max }=952.9 \mathrm{~Hz}$ with the Carrier Frequency $f_{c}=2 \mathrm{GHz}$. The system parameters are: Total number of subcarriers $N=1024$, number of effective subcarriers are 600. The effective bandwidth is $W=5.9 \mathrm{MHz}$. To avoid ISI, the symbol duration is 20 times of the $\tau_{\max }$, i.e., $200 \mu \mathrm{s}$. The $N_{0}=T W=1172$ and $N=L \cdot(2 K+1)=180$. For the OFDM system, the STF basis parameters are chosen to be $W_{0}=90 \mathrm{kHz}$ and $\mathrm{T}_{0}=1 / \mathrm{W}_{0}$ to ensure an orthogonal STF, which correspond to $N_{t}=18, N_{f}=65$. The pilot arrangements are comb-type.

We first justify the first-step of the proposed approach. The simulations are carried out under the assumption that only $10 \%$ of the channel coefficients are nonzero, i.e. D $=18$. The channel matrix is generated from a zero mean Gaussian with variance $\sigma_{D}^{2}$.

Figure 1 depicts the mean square error (MSE) of the channel estimates and the bit error rate (BER) versus the channel signal-to-noise ratio (SNR) in the unit $\mathrm{dB}$. It is seen that both CS-based methods (with $10 \%$ pilots and $25 \%$ pilots) outperform the LS method (with $10 \%$ and $25 \%$ pilots) significantly. The LS and severely underperforms the CS estimator with $10 \%$ pilots even when it itself utilizes $25 \%$ pilots.

Table 2. Two step approach to estimate the HSR NonWSSUS channel.

1. Utilize the delay-Doppler basis to estimate the sparse delay-Dopplerspread function $H_{\alpha}$.

1.1. Use the delay-Doppler basis $U_{p}$ and $H_{\alpha}$ to rebuilt the estimated CIR.

2. Run KF-CS algorithm to track the $H_{a}(n)$ in the sparse domain. Take step 1.1 into account, the Non-WSSUS CIR can be estimated sequentially over time $n$. 


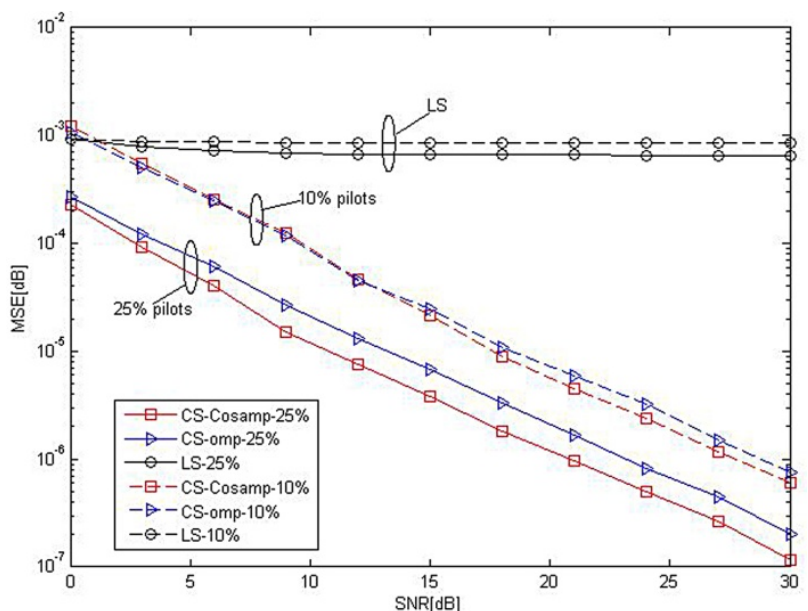

(a)

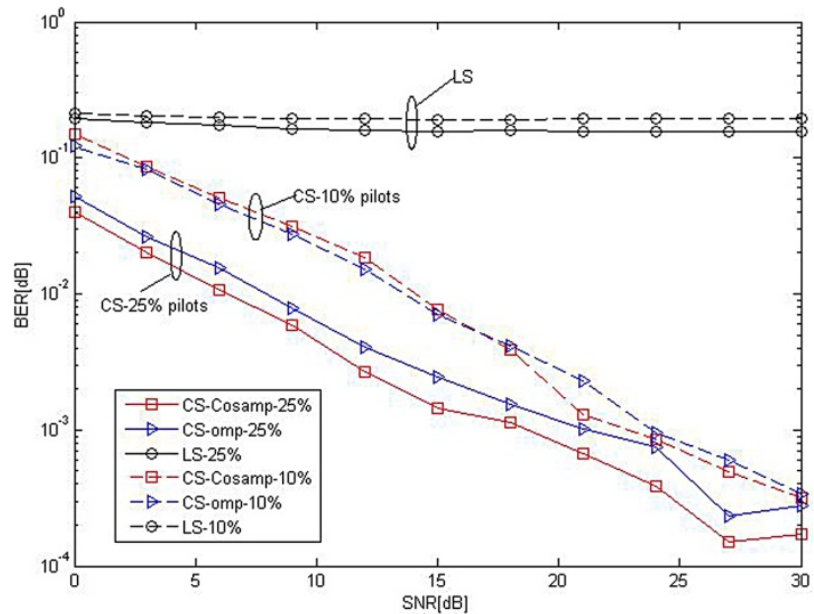

(b)

Figure 1. Performance of CS-based and LS channel estimation. (a): MSE versus SNR; (b): BER versus SNR.

We justify the KF-CS algorithm by using Sequential Compressed Sensing toolbox [18]. Because of the complexity of KF-CS, the large scale data test is very time consuming and easily exceed the hardware memory. In order to promote the efficiency for the practical use, the data symbols are divided into 5 blocks. In each block, there are 234 symbols and 25\% of all are pilot symbols. The simulation results are shown in Figure 2. It can be seen that the KF-CS estimation eventually converges to that of the genie-aided KF (the KF that knows the support at each time) as the time length of $n$ is large enough. In contrast the regular CS methods diverge badly with the increase of time $n$. What is interesting is that the KFCS even outperforms the noiseless CS when $n$ is short (around $n$ is 4) in Figure 2 (Up). This is because that the Kalman filter can track the target signal from noisy environment. This means even the sparse system is static, one can also employ KF-CS to improve the estimation performance.

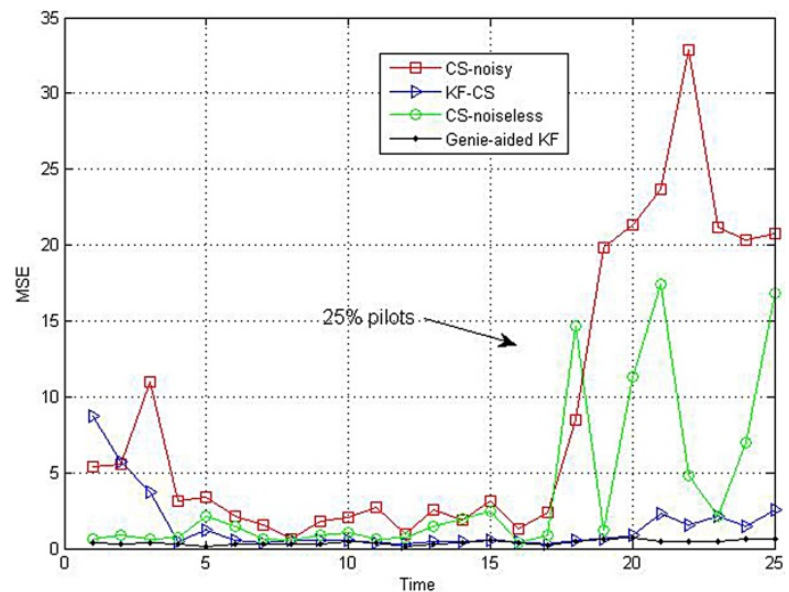

(a)

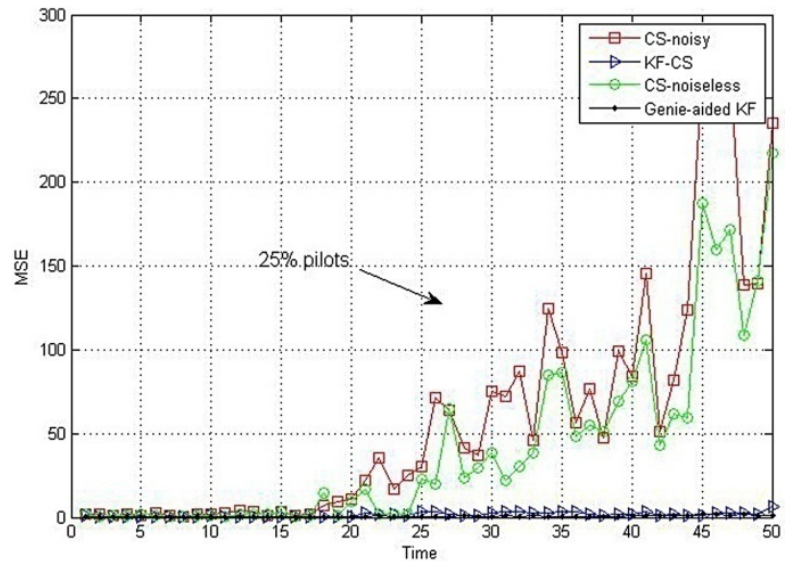

(b)

Figure 2. Performance of KF-CS with the increase of time $n$. (a): time length is 25; (b): time length is 50.

\section{Conclusion}

We have considered the Non-WSSUS channel estimation in the HSR environment with OFDM system. We have proposed a two-step approach to solve this channel estimation problem. In the first step, the channel CIR is estimated by the Compressive Sensing (CS) method by utilizing the sparse delay-Doppler-spread function. In the second the step, the channel is tracked by a reduced order Kalman filter in the sparse domain, and then recovered sequentially. The validation of this approach has been illustrated by numerical experiments.

\section{Acknowledgements}

The authors would like to thank Prof. Namrata Vaswani's research team to share their accomplishment on internet.

\section{REFERENCES}

[1] S. Chen, “China Unveils High-Speed Railways,” 2011. 
http://news.bbc.co.uk/2/hi/8246600.stm

[2] D. Xin., "Record-Breaking Train on Track,” 2010. http://www.chinadaily.com.cn/china/2010-12/04/content11651930.html

[3] L. Liu, et al., "Position-Based Modeling for Wireless Channel on High-Speed Railway under a Viaduct at 2.35 GHz," IEEE Journal on Selected Areas in Communications, Vol. 30, No. 4, 2012, pp. 834-845. http://dx.doi.org/10.1109/JSAC.2012.120516

[4] T. Gao and B. Sun, "A High-Speed Railway Mobile Communication System Based on LTE,” Electronics and Information Engineering (ICEIE), 2010, Vol. 1, pp. 414417.

[5] K. Guan, Z. Zhong and B. Ai, "Assessment of LTE-R Using High Speed Railway Channel Model,” International Conference Communications and Mobile Computing (CMC), 2011, pp. 461-464.

[6] F. Pena-Campos, et al., "Estimation of Fast Time-Varying Channels in OFDM Systems Using Two-Dimensional Prolate," IEEE Transactions on Wireless Communications, Vol. 12, No. 2, 2013, pp. 989-907.

[7] L. L. He, S. D. Ma, and Y. C. Wu, "Pilot-Aided IQ Imbalance Compensation for OFDM Systems Operating Over Doubly Selective Channels,” IEEE Trans on Signal Processing, Vol. 59, No. 5, 2011, pp. 2223-2233. http://dx.doi.org/10.1109/TSP.2011.2112649

[8] H. Hijazi, et al., "Channel Estimation for MIMO-OFDM Systems in Fast Time-Varying Environments," 2010 4th International Symposium on Communications, Control and Signal Processing (ISCCSP), 2010, pp. 1-6.

[9] W. C. Li, and C. P. James, "Estimation of Rapidly TimeVarying Sparse Channels,” IEEE Journal of Oceanic Engineering, Vol. 32, No. 4, 2007, pp. 927-939. http://dx.doi.org/10.1109/JOE.2007.906409
[10] G. Matz, "On Non-WSSUS Wireless Fading Channels," IEEE Transactions on Wireless Communications, Vol. 4, No. 5, 2005, pp. 2465-2478. http://dx.doi.org/10.1109/TWC.2005.853905

[11] M. Jachan and M. Gerald, "Nonstationary Vector AR Modeling of Wireless Channels," Workshop on Signal Processing Advances in Wireless Communications, 2005, pp. 625-629.

[12] W. U. Bajwa, et al., "Compressed Channel Sensing: A New Approach to Estimating Sparse Multipath Channels,” Proceedings of the IEEE, Vol. 98, No. 6, 2010, pp. 10581076. http://dx.doi.org/10.1109/JPROC.2010.2042415

[13] T. H. Eggen, B. B. Arthur and C. P. James, "Communication over Doppler Spread Channels. Part I: Channel and Receiver Presentation,” IEEE Journal of Oceanic Engineering, Vol. 25, No. 1, 2000, pp. 62-71. http://dx.doi.org/10.1109/48.820737

[14] Waheed Uz Zaman Bajwa, "New Information Processing theory and Methods for Exploiting Sparsity Inwireless SystemS,” Ph.D. Thesis, University Of Wisconsin-Madison, 2007.

[15] W. U. Bajwa, A. M. Sayeed and R. Nowak. "Learning Sparse Doubly-Selective Channels," 46th Annual Allerton Conference on Communication, Control, and Computing, 2008, pp. 575-582.

[16] N. Vaswani, "Kalman Filtered Compressed Sensing," 15th IEEE International Conference on Image Processing, 2008. ICIP 2008, 2008, pp. 893- 896.

[17] N. Vaswani, “Analyzing Least Squares and Kalman Filtered Compressed Sensing,” IEEE International Conference on Acoustics, Speech and Signal Processing, 2009. ICASSP, 2009, pp. 3013-3016.

[18] http://home.engineering.iastate.edu/ namrata/research/Se quentialCS.htm 